\title{
Emergency surgery for macrocystic lymphatic malformation in the lung of a neonate
}

\author{
Mamoru Takahashi, MD, ${ }^{\mathrm{a}}$ Norihito Okumura, MD, ${ }^{\mathrm{a}}$ Kotaro Kameyama, MD, ${ }^{\mathrm{a}}$ and Kenji Notohara, MD, \\ Okayama, Japan
}

We report an extremely uncommon case of macrocystic lymphatic malformation found in the lung of a neonate. The patient was a 28 -day-old male neonate with a large cystic lesion occupying the entire lower lobe of the left lung and causing cardiopulmonary dysfunction. Cardiopulmonary status improved soon after emergency surgery comprising left lower lobectomy. The patient has remained well without any signs of recurrence for 48 months after the operation.

\section{CLINICAL SUMMARY}

A 28-day-old male neonate was referred to our hospital after acute onset of dyspnea. Symptoms had been developing for 2 days before admission. In terms of birth history, he was delivered by cesarean section at 36 weeks' gestation as 1 of a set of dichorionic-diamniotic twins. Birth weight was $2580 \mathrm{~g}$. Medical history was otherwise unremarkable. On examination, he had tachycardia, cyanosis, and decreased air entry in the left hemithorax without fever. Functional oxygen saturation was $85 \%$ in room air. Decreased blood pressure required catecholamine infusion. Laboratory data did not show any evidence of infection.

Radiography revealed a large $6 \times 6$-cm lesion with multiseptate, air-filled cysts occupying the left lung. The mediastinum showed marked deviation to the right (Figure 1, $A$ and $B)$. Emergency surgery was indicated with a preoperative diagnosis of congenital cystic adenomatoid malformation.

Anterolateral thoracotomy revealed a distended cystic lesion originating from the left lower lobe and containing no fluid. The upper lobe appeared macroscopically normal. The cystic lesion was completely removed by a left lower lobectomy. Hemodynamic and respiratory status improved soon after the operation. The postoperative course was uneventful.

Histopathologic examination revealed a circumscribed intrapulmonary lesion composed of multipseptate cysts

\footnotetext{
From the Departments of Thoracic Surgery ${ }^{\mathrm{a}}$ and Pathology, ${ }^{\mathrm{b}}$ Kurashiki Central Hospital, Okayama, Japan.

Disclosures: Authors have nothing to disclose with regard to commercial support.

Received for publication Feb 11, 2011; revisions received April 16, 2011; accepted for publication June 7, 2011; available ahead of print July 11, 2011.

Address for reprints: Mamoru Takahashi, MD, Department of Thoracic Surgery, Kurashiki Central Hospital, 1-1-1 Miwa, Kurashiki, Okayama 710-8602, Japan (E-mail: mt10947@yahoo.co.jp).

J Thorac Cardiovasc Surg 2011;142:e133-4

$0022-5223 / \$ 36.00$

Copyright (c) 2011 by The American Association for Thoracic Surgery doi:10.1016/j.jtcvs.2011.06.012
}
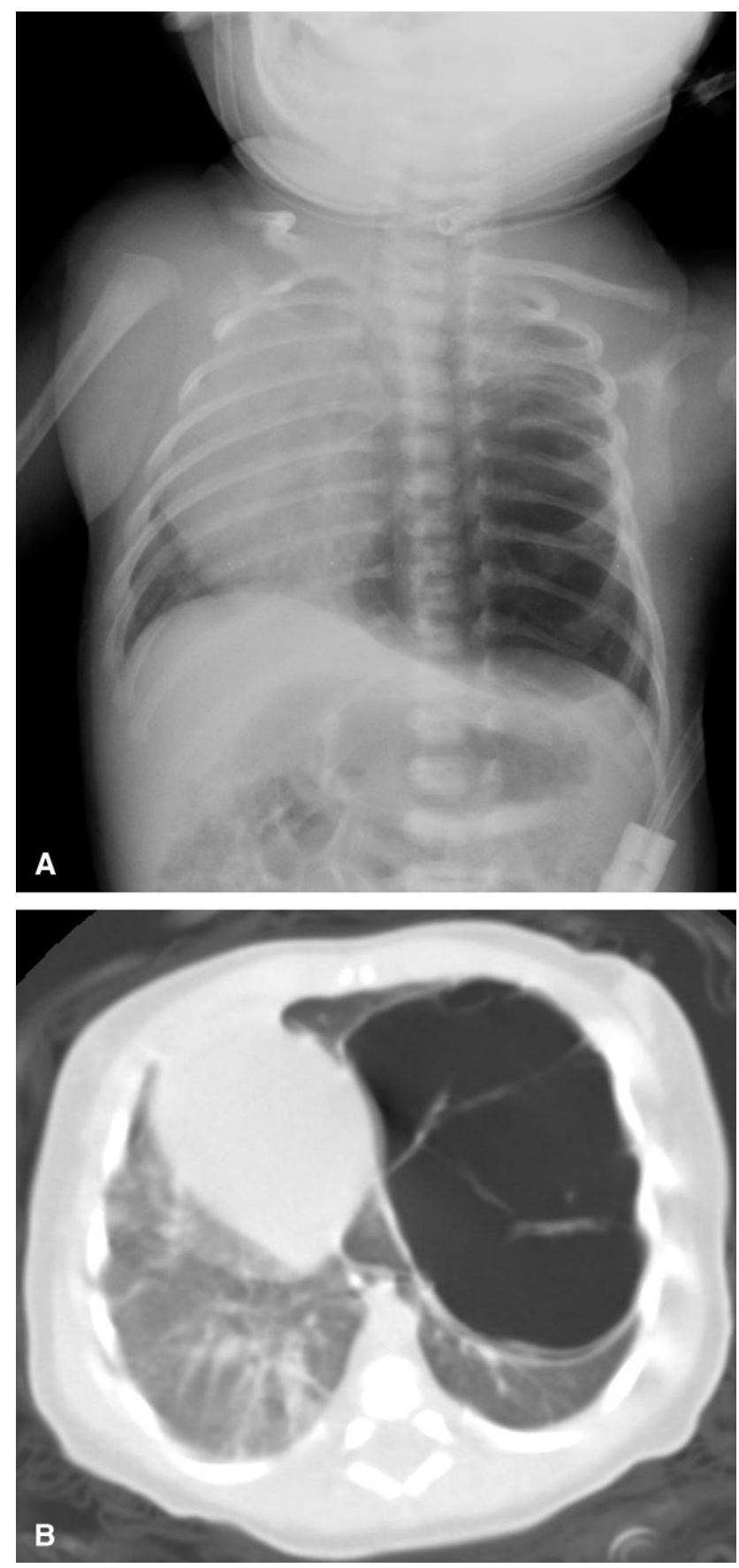

FIGURE 1. A, Chest x-ray film on admission showing a large cyst in the left lung field and mediastinal shift to the right. B, Computed tomographic scan on admission showing a lobulated cyst in the lower lobe of the left lung. The mediastinum was markedly shifted to the right owing to the lesion in the left lung. 


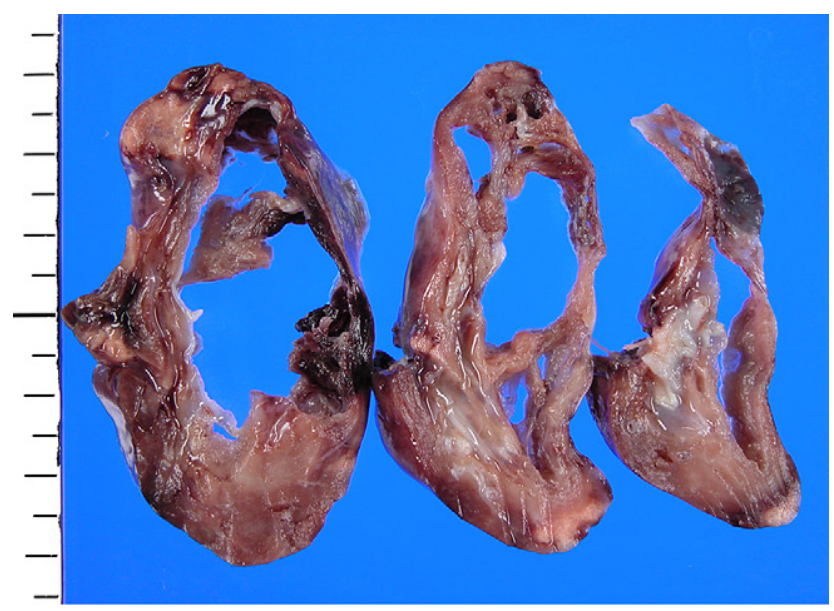

A
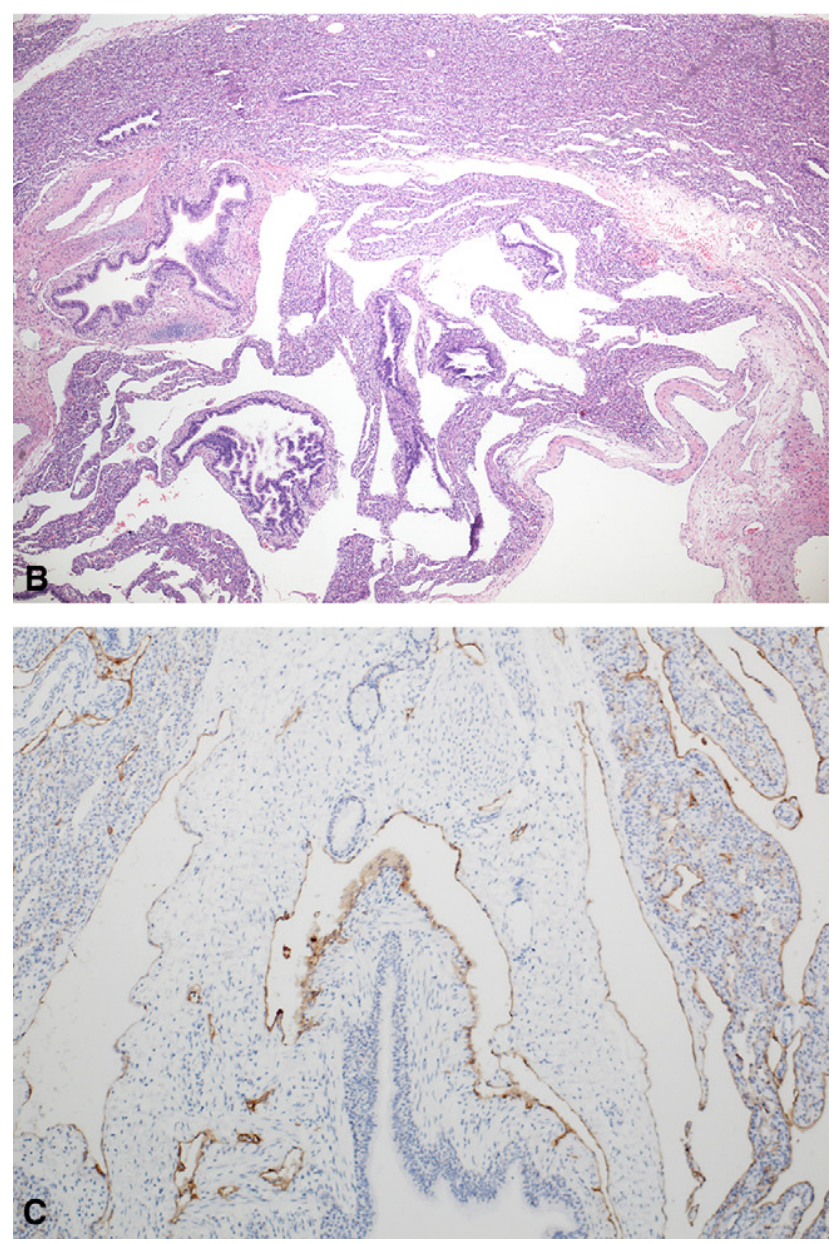

FIGURE 2. A, Gross appearance of the resected lung: a circumscribed intrapulmonary cystic lesion composed of multiple cysts. B, The inner surface of the cysts was lined with flattened cells without atypia. (Hematoxylin and eosin stain; original magnification, $40 \times$.) $\mathrm{C}$, The lining cells are immunoactive with D2-40. (Original magnification, $40 \times$.)
(Figure 2, A). Microscopically, the inner surfaces of the cysts were lined with flattened cells without atypia (Figure 2,B). Immunohistochemical staining of the cell linings yielded positive results for the monoclonal antibody D2-40 (Figure 2, C) and CD31 and negative results for thyroid transcription factor 1 . A diagnosis of lymphatic malformation in the pulmonary parenchyma was established. ${ }^{1}$ The patient has remained well without any signs of recurrence for 48 months after the operation.

\section{DISCUSSION}

Historically known as "lymphangioma" or "cystic hygroma," slow-flow vascular anomalies of the lymphatic system are rare and typically occur in the neck and axilla. Space-occupying lymphatic malformations are extremely uncommon in the pulmonary parenchyma. No more than 20 cases of macrocystic lymphatic malformation in the lung appear to have been reported. ${ }^{2,3}$ Neonatal cases are even less common, with only 1 previous description in which long-term results were not described. ${ }^{4}$ In addition to the rarity, our case was unique in that the patient did not have symptoms until some time after birth. This indicates that cystic malformation of lymphatic channels can expand and become symptomatic, as previously reported. ${ }^{5}$ In this case, we assumed that when the lymphatic malformation eroded the airway after cystic expansion, lymph contents were drained and air became trapped in the lesion, possibly owing to some check-valve mechanism. The inflated lesion then gradually displaced the surrounding normal lung and the mediastinum, leading to cardiopulmonary instability.

In summary, lymphatic malformation in the neonatal lung is extremely uncommon but can result in a lifethreatening situation necessitating emergency surgery. The clinical course in our case suggests that lymphatic malformation in the lung can expand and become symptomatic. The postoperative course to date indicates that emergency lobectomy for a neonate is acceptable.

\section{References}

1. Kahn HJ, Bailey D, Marks A. Monoclonal antibody D2-40, a new marker of lymphatic endothelium, reacts with Kaposi's sarcoma and subset of angiosarcoma. Mod Pathol. 2002;15:434-40.

2. Schulman JM, Christison-Lagay ER, Kozakewich HPW, Boiselle PM, Burrows PE, Fox VL, et al. Macrocystic lymphatic malformation in the pulmonary parenchyma. Ann Thorac Surg. 2009;87:1607-9.

3. Limmer S, Krokowski M, Kujath P. Pulmonary lymphangioma. Ann Thorac Surg. 2008;85:336-9.

4. Milovic I, Oluic D. Lymphangioma of the lung associated with respiratory distress in a neonate. Pediatr Radiol. 1992;22:156.

5. Kavunkal AM, Ramkumar J, Gangahanumaiah S, Parimelazhagan KN, Cherian VK. Isolated mediastinal cystic lymphangioma in a child. J Thorac Cardiovasc Surg. 2007;134:1596-7. 